\title{
Demonstrability of Narrowband Internet of Things technology in advanced metering infrastructure
}

\author{
Liang Wan, Zhijian Zhang and Jian Wang*
}

\begin{abstract}
The deployment cost of advanced metering infrastructure (AMI), one of the key elements for smart grid, often arises from three sub-systems, respectively referred to as data management system, communication network, and smart devices. Among them, the costs of communication network account for a considerable proportion. In smart grid, smart devices are generally fixed in a certain position and schedule reading of interval meter data. They do not only cover smart meters, but also extend gas, water meters, and other sensors measuring light, humidity, and temperature. Many of them are often with battery supply, and this will bring utilization constraints especially in communication network. The deployment needs of reducing cost and the energy-constrained network for an extremely large-scale AMI infrastructure both require optimum communication technologies that consider energy consumption, minimize energy use, simplify network topology, and prolong network lifetime. As an emerging 3GPP radio interface technology specifically designed for low power wide area networks (LPWANs), Narrowband Internet of Things (NB-IOT) inherits from the existing LTE but does many simplifications. Its most prominent features, enhanced coverage and low power consumption, are being pursued for a large-scale AMI communication network. In this paper, we provide a survey of AMI communication, emphasize on the key technologies of NB-loT, analyze the performance of NB-loT network over a real-world, and demonstrate the potential of NB-loT to support and boost AMI in smart grid.
\end{abstract}

Keywords: Advanced metering infrastructure, NB-IOT, LPWAN

\section{Introduction}

Smart grid, the next generation electric grid, is a two-way data communication network which enables the smart integration of conventional power generation, renewable generation, distributed generation, energy storage, transmission, distribution, and demand management [1]. Through automated control and communication technologies, it brings great benefits including the enhanced reliability and responsiveness, higher operational efficiency, more efficient self-management, and better power quality. As one of the key sub-systems of smart grid, advanced metering infrastructure (AMI) is aimed not only to collect all the data and information from smart meters/sensors and consumers, but also to implement control signal and instruction so as to perform accurate

\footnotetext{
*Correspondence: wangjnju@nju.edu.cn

School of electronic science and engineering, Nanjing University, No.163, Xianlin Avenue, Qixia District, 210023 Nanjing, China
}

and effective control actions throughout smart grid [2]. The transmission of these information flows among all the components is completely dependent on the communication network. AMI is usually responsible for smart electricity, but is not limited to. Smart gas, water meters plus other sensors measuring light, humidity, and temperature can also be covered [3] and collectively be called as smart devices. As the main components of the AMI networks, smart meters could play the role of the access points, and the other home area network (HAN) sensors collect and forward the measurement data to smart meters. In an extended AMI network, the number of smart devices may become quite high, even up to a high order of magnitude, and they typically implement information interaction with other components using some combination of wireless and wired communications [4].

Both wireless and wired communications should meet specific requirements, i.e., reliability, latency, bandwidth, and security, depending on AMI tasks, and the cost of 
the deployment is also very critical, especially for the extremely large-scale applications. According to the Electric Power Research Institute [5], the cost associated with the deployment of AMI arises from three sources respectively referred to as data management system, communication network, and smart devices. The costs of communication network and smart devices account for up to $20 \%$ and $45 \%$ respectively, generally including the infrastructure cost, the deployment cost, and all the maintenance costs. For an extremely large-scale AMI, these costs need to be shared among tens of thousands of smart devices, and thus, each smart device's share of the operating expense would be very low. Smart devices distributed in a wide range of areas are generally fixed in a certain position, such as homes, offices, buildings, or fields, and schedule reading of interval meter data. The main features of the smart devices could be summarized as stationary, multiple points, low energy consumption, and extensive deployment. Communication infrastructures for smart meters and meters/sensors like water and gas consumption are very similar in many aspects yet differ in some traits. Smart meters deployed as access points, in need of higher bandwidth, are typically fed from the same electric feed that they are monitoring $[6,7]$. Nevertheless, gas and water meters often send small packets periodically in uplink and receive a small amount of instruction flow in downlink. Furthermore, they often run completely on battery which will bring utilization constraints especially in communication network [8]. Prolonging the network lifetime can reduce expenditure, so it is required to have a minimum battery life expectancy of 12 or 15 years, and even 20 years for some applications [9]. Considering the high cost for energy consumption and battery replacement in extremely large-scale network, smart devices have to be in low cost range and low energy consumption; however, these are quite dependant on the external factors such as communication network, electronic devices, and measured quantity. In this study, we focus on the communication technology. The deployment needs of reducing cost and the energy-constrained network for an extremely large-scale AMI infrastructure both require optimum communication technologies that consider energy consumption, minimize energy use, simplify network topology, and prolong network lifetime.

Low power wide area network (LPWAN) has become an exciting new trend in the development of the wireless communication system since it was first proposed as a class of wireless technology used for machine-to-machine (M2M) or Internet of Things (IoT) communications in 2013. Compared with traditional wireless technologies such as mesh, Zigbee, Wi-Fi, Bluetooth, and cellular, LPWAN has longer lifetime of nodes, lower transceiver chip cost, and larger coverage area, and these main characteristics distinguish itself from other technologies [10]. So far, many of the LPWA technologies have emerged in both unlicensed and licensed markets, such as LTE-M, weightless, SigFox, LoRa, and Narrowband Internet of Things (NB-IoT)[11]. Among them, NB-IoT that was standardized in Release 13 of 3GPP's specification in 2016 is one of the leading emergent technologies. The performance objectives of NB-IoT are to provide high coverage (20 dB improvement compared to GPRS), low device power consumption (a battery life of up to more than 10 years), low delay sensitivity (less than $10 \mathrm{~s}$ ), massive capacity (cellsite sector, at least 52,547 devices), and ultralow device cost (less than \$5) [12]. These enable a high potential for realization of AMI applications. So we establish a set of experimental system based on China Telecom NB-IoT network and commercial cloud platform, choose different data sources, compare the performance of NB-IoT over a real world with the requirements of AMI communication, analyze the feasibility, and introduce the technology to boost AMI applications.

The contributions of the paper are listed as follows: First, most of NB-IoT technology researches are based on the theoretical or simulated analysis, rarely related to the real-world NB-IoT network, so our research fills that gap. Second, AMI usually combines multiple communication technologies, and our research enriches and extends the selectivity of AMI systems which could lower the cost of AMI deployment and support the energy-constrained network. Third, NB-IoT is a new technology; many customers and companies still have many concerns about the NB technology, and our research have demonstrated the potential of NB-IoT to support and boost AMI in smart grid which would contribute to the promotion and application of NB-IoT technology.

\section{AMI communication}

AMI communication system is a crucial component for AMI, and it plays a vital role in meeting the complex potential applications of smart grid. Two-way distributed, real-time or near-real-time, and centralized are the main characteristics of AMI communication [13]. The system is a multi-tier network supported by various existing wired and wireless, unlicensed and unlicensed communication technologies including power line carrier (PLC), copper or optical fiber, cellular, WiMax, Bluetooth, general packet radio service (GPRS), Internet, satellite, Peer-to-Peer, and Zigbee [14]. In the future, fifth generation (5G) network which could provide services for a wide range of machinetype applications would also flexibly support this system [15-17]. AMI information flows over all the sections of the smart grid. Based on different requirements of the wide area network (WAN), neighborhood area network (NAN), and home area network (HAN), a well-designed AMI setup can also be seen as having three different layers, as shown in Fig. 1 [18, 19]. 


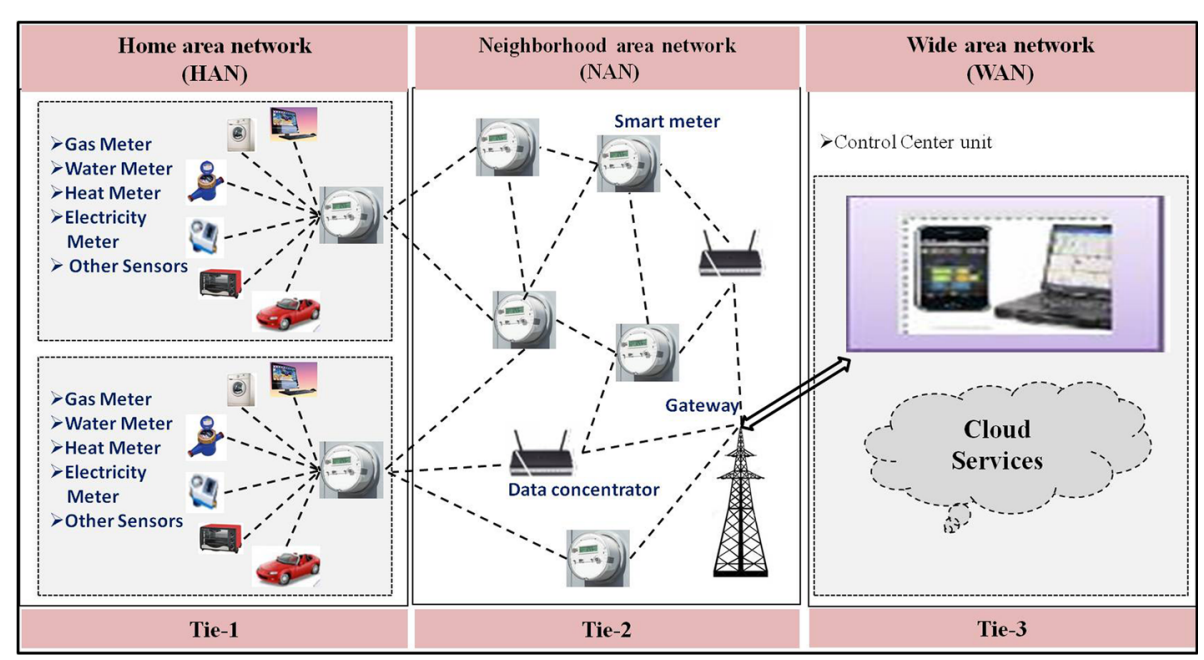

Fig. 1 Multi-tier smart grid communications architecture

\subsection{WAN}

WAN forms the communication backbone of AMI and generally interconnects all the distributed area networks that server AMI system at different locations. To be fully effective, many high bandwidth communications link for AMI communication backbones should be provided, and they would be used to delivery a large number of critical information flow including real-time metering data obtained at the electric devices and command signal between the control center and HAN/FAN. Long-distance and broadband-data rates may be the main requirements in data collection and transmission. Additionally, the latency requirement would become the most vital if the control instructions from the control center must be implemented within a few milliseconds to prevent emergencies. The coverage of WAN would be in order of thousands of square miles while the data rates would be between 10 and 100 Mbps $[19,20]$. These requirements make less competition in selecting communication technologies for WAN, and the fiber communication, cellular networks, WiMAX, satellite communication, and 5G network could be elected as the best candidate technology for WAN.

\subsection{NAN}

NAN can be considered as a bridge which collects data from multiple HANs and transmits the data to a data concentrator, and the command information in the other direction. NAN is a mesh network of smart meters, data collectors, and gateways. Smart meters typically act as the access points or relay nodes, and the backup power is generally not provided for them. NAN could also be called field area network when it is comprised of field electronic devices. There are usually no strict standards for the definition of this network. A single-hop approach is permitted, and also multiple hops approach is allowed to achieve a large coverage, although it would make the network more complex and costly. High scalability which would allow addition of more access points is necessary, and time sensitivity is not much of an issue. NAN requires bandwidth varying from $100 \mathrm{Kbps}$ to $10 \mathrm{Mbps}$, and the coverage distance is up to $10 \mathrm{~km}$. NAN can be implemented over a large number of communication technologies, such as ZigBee, WiFi, PLC, WiMAX, cellular, and Digital Subscriber Line (DSL) [19-22].

\subsection{HAN}

HAN at present is a highly contested space including a large number of different electronic devices (IEDs) that are sensor-based controllers of power system equipments, with a number of communication options. The aim of HAN is to collect the measured data from different applications in a home or a building area. HAN will connect to the access points in NAN. HAN data flow is instantaneous rather than continuous, and devices are typically powered by stored energy, e.g., batteries, so low power consumption, low cost, and simplicity should be necessarily considered [23]. The communication technologyrequired latency will be in the range of $2-15 \mathrm{~s}$ for some types of data traffic, and reliability requirements will be in the range from 99 to $99.99 \%$ [24, 25]. HAN requires bandwidth varying from 10 to $100 \mathrm{Kbps}$ for each device, and the coverage distance is about $100 \mathrm{~m}$. Given the above requirements and considering the short distances among nodes that enable low power transmission, wireless technologies are the dominant solutions for HAN. These technologies include $2.4 \mathrm{GHz}$ WiFi, 802.11 wireless networking protocol, ZigBee, and HomePlug [19-21]. 
There are obvious differences in the coverage range and data rate for WAN/NAN/HAN. Wireless technologies are gaining more attraction than wired technologies, due to low installation cost, rapid deployment, mobility, and flexibility. Table 1 presents the current wireless communication technologies that can support AMI applications. AMI communication system is not one type of communication technology, and actually, no single communication technology or network structure can cost-effectively support the system to different area applications which are deploying urban, suburban, rural, and remote locations, small and large buildings, houses, basements, etc. [24]. For example, the cellular networks could provide high data rate up to $100 \mathrm{Mbps}$ and coverage range up to $50 \mathrm{~km}$, with advantages such as wide deployment, long-distance connection, high rates of data transfer, and telecommunications level security. It seems that cellular network is great for each layer, but actually, it is poor for some applications which have huge amounts of smart devices in HAN/NAN due to deployment cost, energy consumption, difficulty of devices certification, and limited capacity. Some utility

Table 1 Wireless communication technologies that support AMI applications

\begin{tabular}{|c|c|c|c|c|}
\hline Technology & $\begin{array}{l}\text { Standard/ } \\
\text { protocol }\end{array}$ & $\begin{array}{l}\text { Max. theoretical } \\
\text { data rate }\end{array}$ & $\begin{array}{l}\text { Coverage } \\
\text { range }\end{array}$ & $\begin{array}{l}\text { Network } \\
\text { layer }\end{array}$ \\
\hline Z-Wave & Z-Wave & $40 \mathrm{Kbps}$ & $\begin{array}{l}\text { Up to } \\
30 \mathrm{~m}\end{array}$ & HAN \\
\hline Bluetooth & 802.15 .1 & $721 \mathrm{Kbps}$ & $\begin{array}{l}\text { Up to } \\
100 \mathrm{~m}\end{array}$ & $\mathrm{HAN}$ \\
\hline \multirow[t]{2}{*}{ ZigBee } & ZigBee & $250 \mathrm{Kbps}$ & $\begin{array}{l}\text { Up to } \\
100 \mathrm{~m}\end{array}$ & HAN/NAN \\
\hline & ZigBee Pro & $250 \mathrm{Kbps}$ & $\begin{array}{l}\text { Up to } \\
1600 \mathrm{~m}\end{array}$ & HAN/NAN \\
\hline WLAN & $802.11 x$ & 2-600 Mbps & $\begin{array}{l}\text { Up to } \\
100 \mathrm{~m}\end{array}$ & HAN/NAN \\
\hline WiFi & $802.11 x$ & 2-600 Mbps & $\begin{array}{l}\text { Up to } \\
100 \mathrm{~m}\end{array}$ & HAN/NAN \\
\hline WIMAX & 802.16 & $75 \mathrm{Mbps}$ & $\begin{array}{l}\text { Up to } \\
50 \mathrm{~km}\end{array}$ & NAN/WAN \\
\hline \multirow[t]{5}{*}{ Cellular } & GPRS & 14.4 Kbps & $\begin{array}{l}\text { Up to } \\
50 \mathrm{~km}\end{array}$ & NAN/WAN \\
\hline & $2.5 G$ & $144 \mathrm{Kbps}$ & $\begin{array}{l}\text { Up to } \\
50 \mathrm{~km}\end{array}$ & NAN/WAN \\
\hline & $3 G$ & $2 \mathrm{Mbps}$ & $\begin{array}{l}\text { Up to } \\
50 \mathrm{~km}\end{array}$ & NAN/WAN \\
\hline & LTE & $14 \mathrm{Mbps}$ & $\begin{array}{l}\text { Up to } \\
50 \mathrm{~km}\end{array}$ & NAN/WAN \\
\hline & $4 G$ & 100 Mbps & $\begin{array}{l}\text { Up to } \\
50 \mathrm{~km}\end{array}$ & NAN/WAN \\
\hline Satellite & Satellite & $1 \mathrm{Mbps}$ & $\begin{array}{l}100- \\
6000 \mathrm{~km}\end{array}$ & WAN \\
\hline
\end{tabular}

companies have mixed short-range connectivity communications (ZigBee, Bluetooth, WiFi, etc.) and mesh technology together to deploy smart devices in AMI. The solution presents a positive characteristic for supporting many applications, but it also causes some disappointments. First, this solution brings an increase in cost. Many devices are hard to reach when linking the endpoint; thus, they need multiple hops and additional relays. Meanwhile, mesh devices must constantly receive and repeat its neighbor's RF signals that will increase energy consumption in more dense scenarios, and also bring utilization constraints for battery supply devices. Another disadvantage is that this solution increases the complexity of network. To make all devices reachable by the mesh, a large number of relays should be added which would complicate network topology, and various complex network protocols also would be used in devices. All of these would cause the probability of failure becoming unpredictable and make the network management complicated. In future smart grid, as the scale of AMI communication network extends exponentially, the coverage will spread over a considerably large area, e.g., thousands of kilometers in dimension, and the connectivity solution for smart devices will become a very important issue and a highly contested space. At the same time, this will bring numerous challenges and opportunities to all the communication technologies.

\section{NB-loT technology}

Narrowband Internet of things (NB-IoT) was introduced as a new radio interface to the specification 3GPP Rel.13 in 2016. It is designed to support a massive number of devices, low data rate, low device power consumption, an extreme coverage, and ultralow device cost. NB-IoT is new narrower radio interface, but it inherits basic functionalities from the LTE system and can coexist with GSM and LTE networks [26]. The parameters of NB-IoT technology are shown in Table 2. NB-IoT utilizes a frequency

Table 2 The parameters of NB-loT technology

\begin{tabular}{ll}
\hline Parameters & Values \\
\hline Spectrum & Licensed LTE bandwidth \\
Modulation & QPSK \\
Channel bandwidth & $180 \mathrm{kHz}$ \\
System bandwidth & $180 \mathrm{kHz}$ \\
Peak data rate & $\mathrm{DL}: 234.7 \mathrm{kbps}$; UL: $204.8 \mathrm{kbps}$ \\
MCL (maximum coupling loss) & $164 \mathrm{~dB}$ \\
Energy efficiency & More than 10 years battery \\
Interference & Low \\
Peak current & $120-300 \mathrm{~mA}$ \\
Sleep current & $5 \mu \mathrm{A}$
\end{tabular}


bandwidth of $180 \mathrm{kHz}$, which corresponds to one physical resource block (PRB) in LTE transmission. The downlink consists of narrowband physical broadcast channel $(\mathrm{NPBCH})$, narrowband physical downlink control channel (NPDCCH), narrowband physical downlink shared channel (NPDSCH), narrowband reference signal (NRS), narrowband primary synchronization signals (NPSS), and narrowband secondary synchronization signals (NSSS), while the uplink includes Narrowband Physical Uplink Shared Channel (NPUSCH) and Narrowband Physical Random Access Channel (NPRACH). These channels are based on existing LTE channels with necessary modifications to fit into the narrow bandwidth used by NB-IoT [27]. Enhanced coverage and low power consumption are the most prominent features of NB-IoT. The former one is decided by key technologies including narrowband (NB), signal repetition (SR), and low frequency (LF), whereas the latter one is mostly achieved through extended discontinuous reception (eDRX) and power-saving mode (PSM) [28].

\subsection{NB}

Narrowband technology can provide a higher power spectral density (PSD) for NB-IoT with respect to LTE. NB-IoT is kept to a bandwidth of $180 \mathrm{kHz}$ corresponding to one PRB of LTE network for both downlink and uplink [29]. In the downlink, NB-IoT is based on orthogonal frequency-division multiplexing (OFDM) with $15 \mathrm{kHz}$ subcarriers spacing and each OFDM symbol consists of 12 subcarriers, while single-tone and multi-tone operations are supported in the uplink. $3.75 \mathrm{kHz}$ and $15 \mathrm{kHz}$ subcarrier spacing can be both used in single-tone operation, and $15 \mathrm{kHz}$ is supported with single-carrier frequency-division multiple access (SC-FDMA) [29, 30]. This means each OFDM symbol has 12 or 48 subcarriers. NB-IoT inherits LTE, and the node transmission power is still $43 \mathrm{dBm}$ which is the same as in LTE. It results in a better PSD that would improve the received signal-to-noise ratio (SNR) at least by a factor of about
$11 \mathrm{~dB}$, so it allows the node to reach higher covered distance compared to the GSM case under the same conditions.

\subsection{SR}

In order to achieve enhanced coverage, multiple SR is supported for uplink and downlink data transmissions in NB-IoT [31]. With the help of multiple SR, the devices with low power that are in weak coverage condition can boost the signal strength. The number of SR depends on the coverage enhancement level required by a user equipment (UE) [32]. A UE decides its coverage enhancement level based on the downlink measurement, e.g., reference signal received power (RSRP) threshold set by the network. Theoretically, NPDCCH and NPDSCH are allowed to be up to 2048 SR, whereas NPUSCH and NPRACH support a maximum of 128 SR [31-33]. This would obtain additional $33 \mathrm{~dB}$ and $21 \mathrm{~dB}$ gains in terms of SNR. However, with the increase in the number of SR, the data rate would reduce rapidly. Considering the data rate and cell capacity under the edge scenario over a real world, the number of SR is 16 for the uplink, and a maximum 512 bytes of one packet is supported in China Telecom NB-IoT network.

\subsection{LF}

NB-IoT can be deployed in three operation modes: standalone, guard-band, and in-band [32], as shown in Fig. 2. Stand-alone operation, which can be deployed within one or more GSM carriers by telecom operations, is a popular model, and academically, it can utilize any available spectrum. But operations must avoid conflicting with exciting LTE system and utilize the available spectrum which can be more robust and reliable for long-distance transmission. Compared with high frequency, LF like Sub-1GHz experience less attenuation and multipath fading caused by obstacles and dense surfaces like walls. In China, NBIoT has been deployed commercially based on $850 \mathrm{MHz}$ and $900 \mathrm{MHz}$.

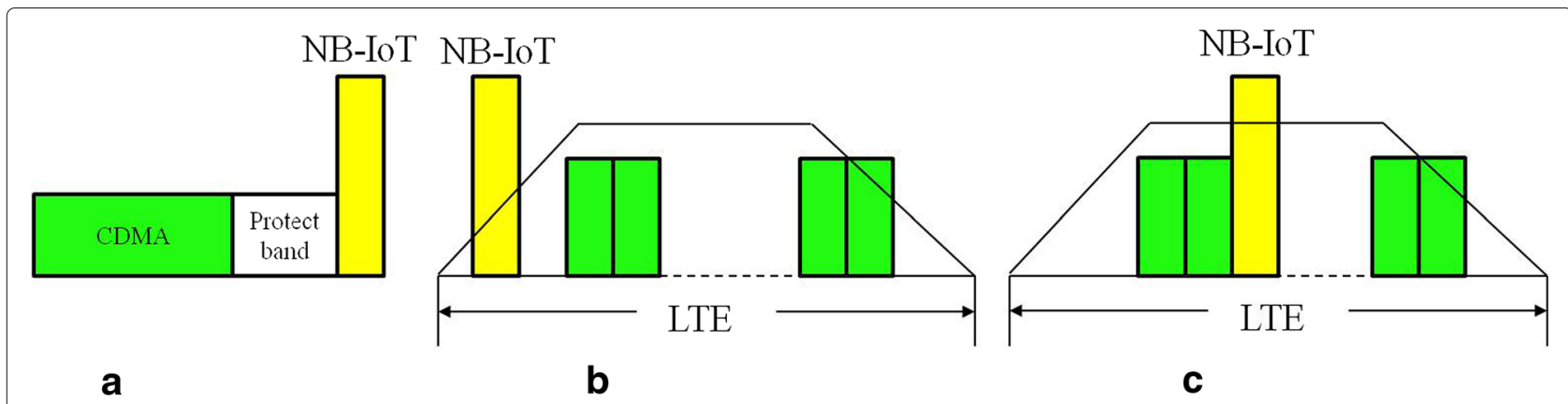

Fig. 2 NB-IoT operation modes. NB-IoT can be deployed in three operation modes: stand-alone, guard-band, and in-band. a Stand-alone, b guard-band, and $\mathbf{c}$ in-band 


\section{4 eDRX}

Discontinuous reception (DRX) is a discontinuous reception technology of downlink control channel which is based on radio resource control (RRC). For the UE set to a DRX cycle in idle mode, the control channel for paging could be monitored during a paging transmission window (PTW). Each DRX is divided into two parts: PRC_connected and PRC_IDLE state [29]. The UE does not connect with the base station all the time only during the PRC_connected state which will be sustained just for $1 \mathrm{~ms}$ per DRX cycle. In PRC_connected state, the UE can monitor the downlink channel. The PRC connection may be suspended and release during idle state, but the information that is required to resume the RRC connection including access stratum context and resume ID has been stored in UE and $\mathrm{eNB}$, and PRC connection can be established in the next monitoring time. DRX is possible to reduce energy consumption based on the DRX cycle. As the DRX cycle becomes larger, the average energy consumption decreases and the period is up to $2.56 \mathrm{~s}$ in LTE [28]. eDRX-inherited DRX is proposed to achieve lower power consumption in NB-IoT. In eDRX, a long DRX cycle is supported, and it is extended beyond 10.24 s with a maximum value of $10,485.76 \mathrm{~s}$ [30]. Without a doubt, the long DRX cycle increases the response time, thus compromising the customer experience. Figure 3 shows the maximum period of DRX and eDRX along with the UE power state.

\subsection{PSM}

PSM is introduced as a more power-efficient mode than eDRX in NB-IoT, presented in Fig. 4. In PSM, the UE is permitted to close signal transceiver and AS function after entering the idle state for a period of time. The UE could not be reachable by the network but remains registered, if it has been in the power-off or sleep mode, and will wake up only when there is MO data to send or after tracking area update (TAU) timer expiration. TAU timer decides the PSM interval which can be a maximum of $310 \mathrm{~h}$, while the period of the idle state depends on the active timer within the range of 0 to $255 \mathrm{~s}$ [33]. PSM is very conducive to the deployment of applications whose devices with batteries are not required to send packets frequently and receive a packet from the network sporadically and the downlink latency of the network could be ignored.

\section{Experiments and results}

\subsection{Experimental setup}

Communication and control are the fundamental building modules of the smart grid. Communication provides the channel for exchanging information between smart devices and control components. The sensing data from smart devices would be delivered to the control components, and the instructions generated from control components would transmit to corresponding elements in smart grid resulting in accurate actions. So we built up an experimental system based on real-world NB-IoT network to verify whether the NB-IoT technology was robust enough to boost AMI applications in smart grid. The measurements took place in different locations on Xianlin Campus of Nanjing University, China. The case's network architecture is shown in Fig. 5. Table 3 describes the components of the network. The commercial cloud platform OceanConnect and NB-IoT eNBs both belong to China Telecom. To fully demonstrate the reliability and adaptability of the NB-IoT network, we selected two different types of data sources for the NB-IoT end devices in experimental scenarios. The first type was the packets

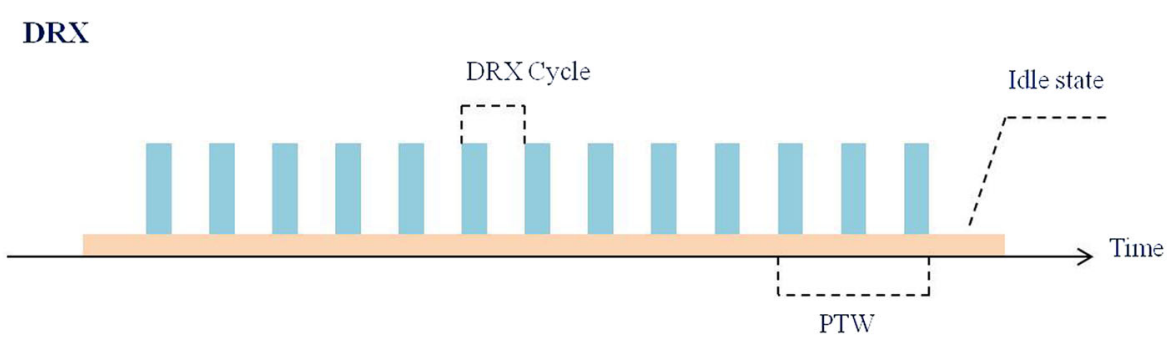

eDRX

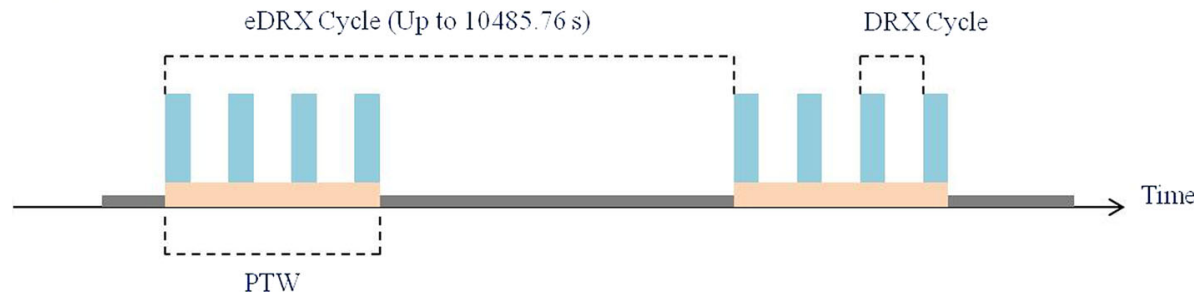

Fig. 3 User equipment (UE) power state : DRX and eDRX. The maximum period of DRX and eDRX along with the UE power state 


\section{PSM}

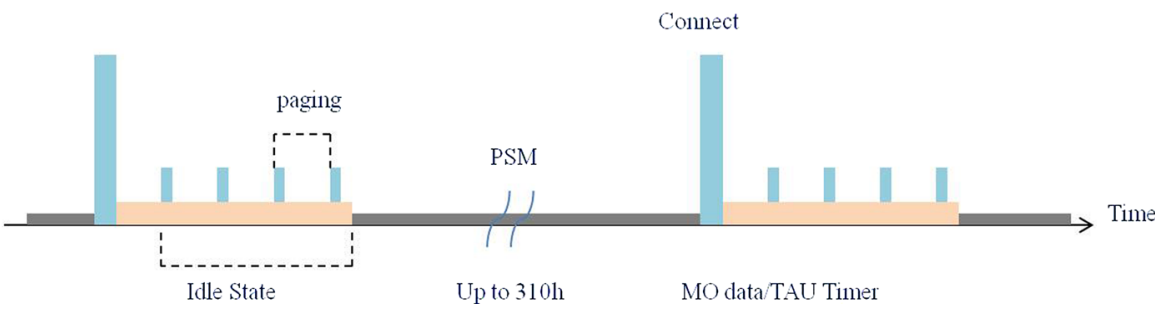

Fig. 4 User equipment (UE) power state : PSM. A more power-efficient mode than eDRX in NB-IOT

of 30 bytes which was generated from a serial port of laptops, and the second type was the packets of more than 100 bytes that were from the concentrator of one automatic meter reading system. The automatic meter reading system took the DL/T645 protocol as the standard for multifunction energy meter communication protocol, and Q/GDW376.1-2009 protocol as the standard protocol for the communication between the main station and the concentrator.

\subsection{Results}

The performance results presented in this section are extracted from our experimentation campaign in Xianlin Campus of Nanjing University. They were measured in terms of coverage, reliability, latency, and battery life, and security was also analyzed in the end.

\subsubsection{Coverage}

NB-IoT network achieves a maximum coupling loss $20 \mathrm{~dB}$ higher than the existing LTE, and three coverage enhancement levels are defined to cope with different radio conditions [32]. When the device in an idle state has a small data packet to transmit, the device has to determine its coverage level based on the reference signal received power (RSRP). Thus, the coverage analysis by considering the RSRP of the devices deployed in outdoor, indoor, and deep indoor environments was reported to investigate the coverage performance in different propagation environments. Figure 6 shows the RSRP of 81 selected test points. The threshold was set to $-97 \mathrm{dBm}$, and the coverage was $100 \%, 100 \%, 95 \%, 5 \%$, corresponding to outdoor, indoor (3rd, 4th, and 5th floors), indoor (1st and 2nd floors), and deep indoor (basements). The results were basically consistent with those reported by the utility company. It means that the real-world NB-IoT network can provide sufficiently good indoor and outdoor coverage to support the connected devices, but for deep indoor, the deviceperceived signal is weak at present and it still has a lot of work to do. To solve this problem, the utility companies are working on the design of indoor distribution systems. Actually, for some scenarios, the deployment of small cells or other local data aggregators may also be a good option.

\subsubsection{Reliability}

Communication reliability is extremely important for the network to be reliable for correct and timely message transmitting, and it is affected by plenty of possible factors. In this study, packet loss rate (PLR) was used to be an indicator to quantify the reliability performance. According to the relationship among the network components, the PLR was counted separately in two parts. The first part was the statistics from each device to OceanConnect platform, and the second is from OceanConnect

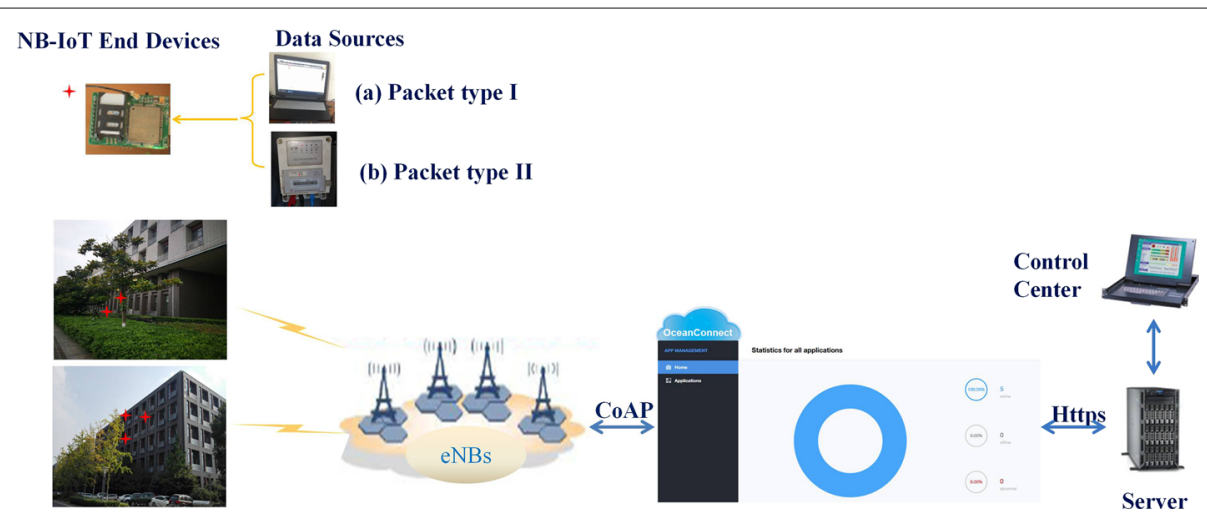

Fig. 5 Network architecture over a real-world environment based on China Telecom NB-loT network. The case's network architecture 
Table 3 Network configure

\begin{tabular}{ll}
\hline Component & Description \\
\hline Data source & (a) Packet type I, 30 bytes, generated from a \\
& serial port of laptops. (b) Packet type II, more \\
& than 100 bytes, generated from \\
& concentrator of the automatic meter \\
& reading system. \\
& Include five end devices, each one is an \\
integrated module and it operates in & stand-alone modes. The integrated module \\
bases on Boudica120 chip, the operating \\
frequency is 850 MHz. \\
Commercial cloud platform, mainly in \\
charge of transparent forwarding data. \\
In charge of collecting data from cloud \\
platform and sending commands to the \\
NB-loT end devices. \\
Bases on China Telecom NB-loT network \\
which have covered most of cities in China. \\
At present, for one pack from the endpoints, \\
the max size of 512 bytes could be \\
supported. \\
(a) Https establishes secure communication \\
between cloud paltform and local server. (b) \\
Constrained application protocol (CoAP), a \\
specialized Internet application protocol for \\
constrained devices which enables the end \\
devices to communicate with Internet. \\
\end{tabular}

platform to local server. In scenario 1, the first type of data source was transmitted. During the measuring period, five devices were installed in the corner of different floors. Two of them sent a packet of 30 bytes every $5 \mathrm{~min}$, total 4032 packets, and the other three sent the same packet every $3 \mathrm{~min}$, total 6720 packets, in 14 days. The first PLR of the devices was $0 \%$. OceanConnect platform was used to transparently forward packets received from the devices, and the second PLR was $99.7 \%$. Actually, retransmission was adopted for every received packet in forwarding. The result of measurements is shown in Fig. 7. In scenario 2, the NB-IoT end devices were responsible for transmitting the data of the concentrators to OceanConnect platform. These data followed the Q/GDW376.1-2009 protocol, and the size of one packet could be up to 112 bytes. Every device sent one packet every 5 min, total 4032 packets, in 14 days. The first PLR of the devices was $0 \%$, and the second PLR was $99.8 \%$. Figure 8 showed the result of measurements. These performances show NB-IoT network is reliable enough to support different AMI applications deployed in outdoor and indoor environment.

\subsubsection{Latency}

Latency defines the maximum time during which a particular message should reach its destination through NB-IoT network. Network topology directly affects the latency, and the data rate supported by NB-IoT also dictates the speed of information exchange. NB-IoT can be considered as a near-real-time network, and it targets latencyinsensitive applications, while it still expects the exception report, latency of $10 \mathrm{~s}$ or less is required for $99 \%$ of the devices [34]. In our experiment, the latency between the devices and OceanConnect platform describing the performance of NB-IoT was discussed. In uplink, the latency was less than $2 \mathrm{~s}$ for each packet (30 bytes). But for the downlink, it had a close relationship with the access point names (APNs) which defined whether the DRX/eDRX/PSM mode was enable, and the parameters

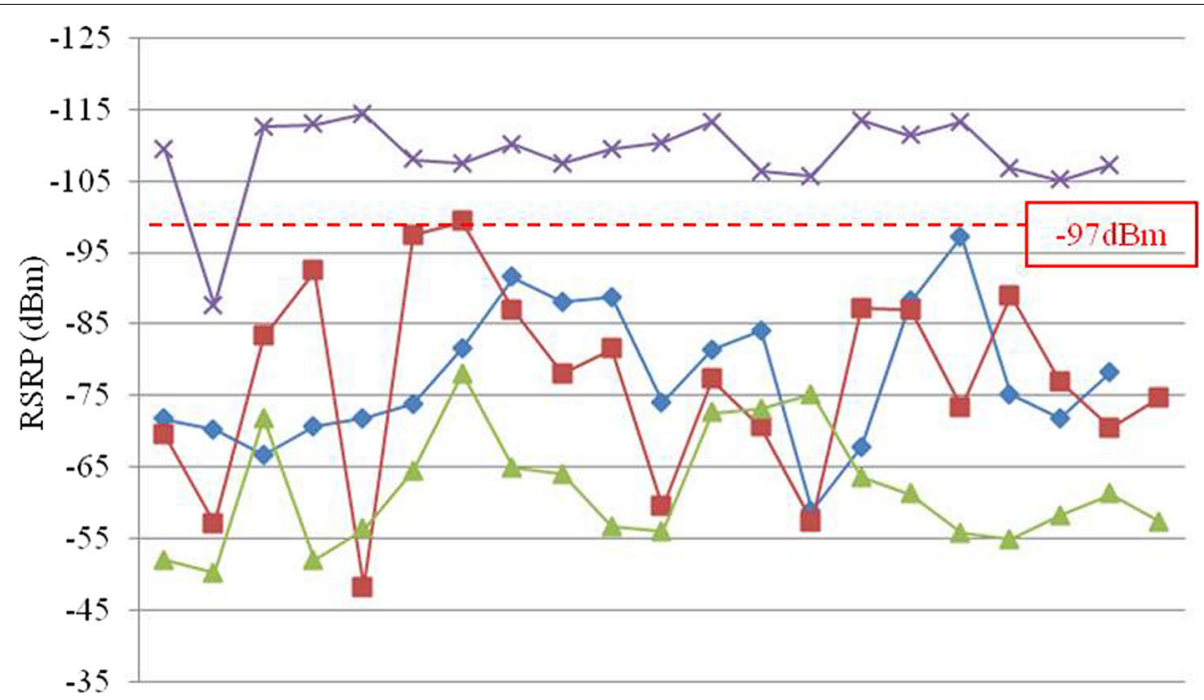

Fig. 6 The RSRP of the devices in different propagation environment. The RSRP of 81 selected test points in our measurements. Legend: the RSRP of indoor (3rd, 4th, and 5th floor) test points (blue); the RSRP of indoor (1st and 2nd floor) test points (red); the RSRP of outdoor test points (green); the RSRP of deep indoor test points (basement) (purple) 


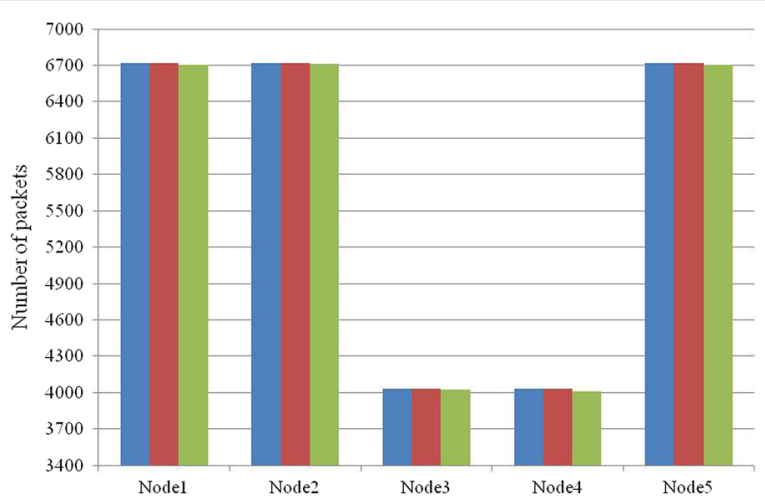

Fig. 7 Results of measurements in scenario 1. The results of measurements in scenario 1. Legend: number of node transmitted packets (blue); number of platform received packets from each node (red); number of server received packets from each node (green)

of different timers, such as active timer and the driven time of the packets, were also an important element. For example, when the device was set to APN_CTNB, the latency would be less than $2 \mathrm{~s}$ if OceanConnect platform replied to a message received within less than $20 \mathrm{~s}$. Otherwise, the message would not be received because the device had been in PSM mode. For AMI applications, the uplink latency could be acceptable for smart devices and data collectors, but the downlink latency may affect the execution of instructions coming from the server if the APN is not suitable. For instance, if we select NB-IoT as the communication technology between the concentrators and the main station, the NB-IoT devices should not be set to APN_CTNB, due to the requirements of Q/GDW376.1-2009 protocol.

\subsubsection{Battery life}

NB-IoT device is expected to achieve a battery life of more than 10 years at maximum coupling loss $(164 \mathrm{~dB})$ using battery capacity of $5 \mathrm{Wh}$, if the device transmits periodically small packet with a daily report of total 200 bytes [30]. PSM and eDRX are the key technologies of NB-IoT, and the long battery life is at the expense of real-time performance. To meet the requirements of different applications such as monitoring or control, operators have deployed multiple optional access point

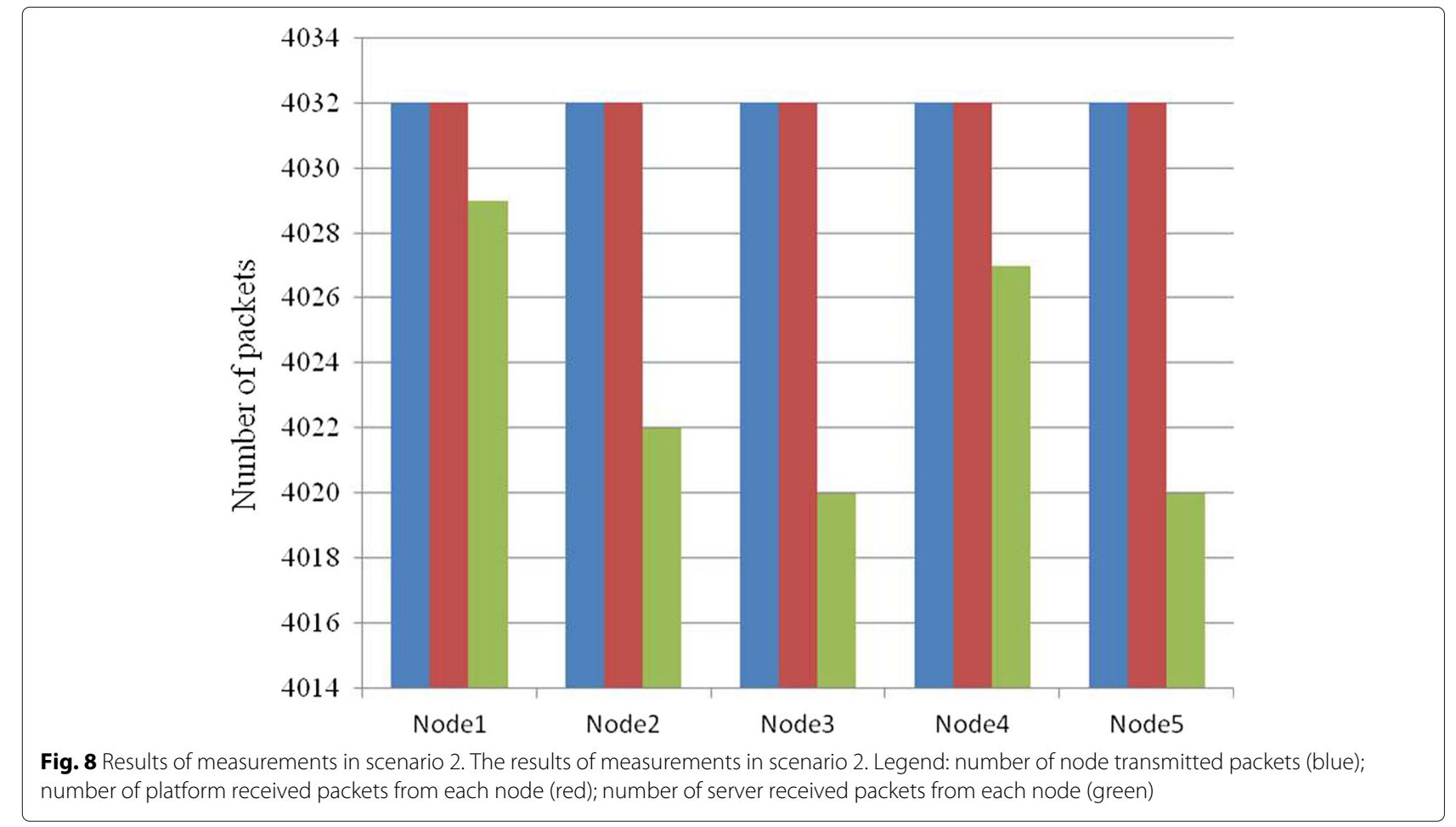


names (APNs) which could make a balance between battery life and transmission requirements. In this study, two APNs, CTNB and PSMF.EDRXC.CTNB, were set for the NB-IoT devices. Figure 9 shows the device current consumptions were $0.03 \mathrm{~mA}$ (PSM), $333 \mathrm{~mA}$ (TRANSMIT), $70.19 \mathrm{~mA}$ (RECEIVE), and $5.93 \mathrm{~mA}$ (IDLE) in CTNB where PSM is enable but eDRX is not. PSMF.EDRXC.CTNB where PSM and eDRX were enable is shown in Fig. 10; the device current consumptions were $0.03 \mathrm{~mA}$ (PSM), $345 \mathrm{~mA}$ (TRANSMIT), $73.22 \mathrm{~mA}$ (RECEIVE), $0.05 \mathrm{~mA}$ (PRC_IDLE), and $66.15 \mathrm{~mA}$ (PRC_CONNECTED). Based on the measurement, the 10-year battery life objective can be achieved or exceeded for daily reporting. Considering millions of smart devices, the deployment cost may decrease in the order of magnitude.

\subsubsection{Security}

According to the Electric Power Research Institute (EPRI), network security is a critical issue for both smart grid and AMI. Especially, as the smart devices increase exponentially and the scale of smart grid expands, the network will cover a considerably large area and security issues will become more prominent. Security concerns might be increased due such factors as the transmission of critical applications and information over a long distance among different components using wireless technologies, as well as storing data in various locations such as terminals, access points, aggregation points, or cloud platforms deployed in different area. NB-IoT inherited basic functionalities from the LTE/4G system, used licensed spectrum, and established on the existing infrastructure with a software or hardware upgrade reusing and upgrading.
Hence, the utility companies must consider appropriate security to the complete system including the core network; available security algorithm has been introduced and the security has been defined as the telecommunications level.

\section{Discussion}

Since the first NB-IoT commercial network was opened in the small town of Hongshan in Wuxi, China, NBIoT technology has become the main topic of fervent research and development at both commercial and academic level. Now, the utility companies including China Telecom, China Unicom, and China Mobile have completed pre-standard pilot work, and the NB-IoT network has operated in all over the country. The network performance has improved significantly, compared with our previous studies whose results for the performance analysis was reported in [35] during the initial stage of network operation. This technology solution has led to success in many commercial applications in China, such as smart parking, intelligent building, and water/gas metering. It seems to be very attractive to AMI applications due to its good performance as discussed in previous section and its high degree of deployment. Additionally, another three issues concerned by the customers and companies should also be considered.

Firstly, NB-IoT technology inherits LTE/4G, and the exciting base stations with upgraded hardware or software are utilized. Therefore, its network connection and distance converge depend on the availability of cellular services. Unfortunately, cellular services are badly dependent on the infrastructure. The weaker resilience against natural disasters, like tsunami, hurricane, and cyclones,

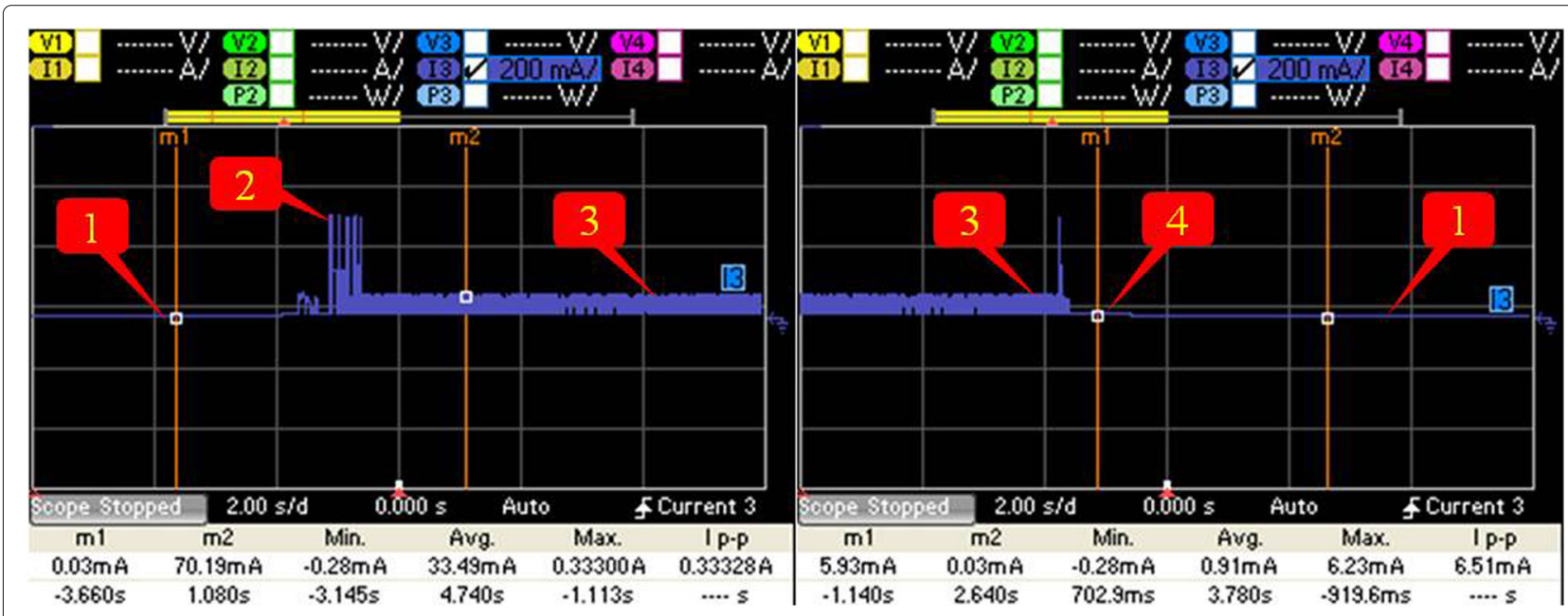

Fig. 9 Device current consumptions in CTNB, measured by Agilent N6705B. The device current consumptions in CTNB, measured by Agilent N6705B. Legend: (1) PSM; (2)TX; (3) RX/active mode transitions; ( 4) Idle 

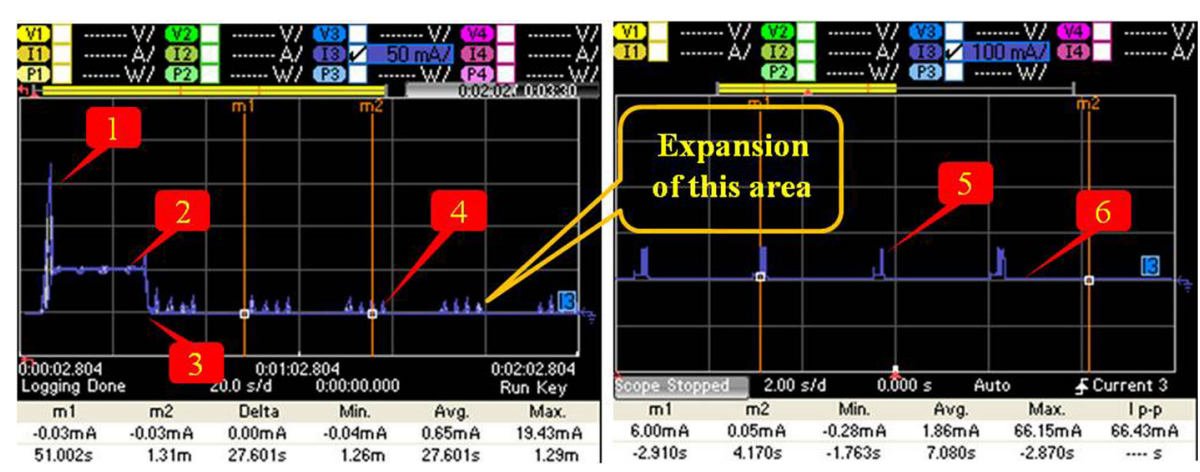

Fig. 10 Device current consumptions in PSMF.EDRXC.CTNB, measured by Agilent N6705B. The device current consumptions in PSMF.EDRXC.CTNB, measured by Agilent N6705B. Legend: (1) TX; (2) RX/active mode transitions; (3) PSM; (4) eDRX; (5) eDRX-CONNECTED; (6) eDRX-IDLE

or man-made, like terrorist attack, massive power failure, more time of recovering from emergencies caused by cellular services sharing with mobile customers, and current inadequate maintenance mechanisms of utility companies would make some customers be hesitant to rely on NB-IoT network at the present stage.

Secondly, once the needs of the NB-IoT service to be deployed are specified, the devices would provide accurate readings automatically at requested time intervals to access points, data collectors, or aggregation nodes. The customers should pay a fee to the utility companies for data collection, management, and analysis, so the billing method would be particularly important. If the fee for individual connection is high and the cost is expensive, the performance objective of low cost would not be achieved and it is clear that the introduction of NB-IoT technology would be meaningless.

Thirdly, the data from smart metering networks generally reveal a wealth of information that linked with individuals or companies; thus, its privacy is important to the users' acceptance. The data interval collection disseminated to different components through NB-IoT network enables the utility companies easily control the information management process and further gives rises to the data privacy problem. The privacy of data concerns the security of itself, and users would need to be reassured that their data is secure. For NB-IoT network, some users express their concerns that their data may be intentionally leaked and even used for purposes beyond energy efficiency by the network maintainers.

Actually, to reassure potential customers and successfully occupy the market, the utility companies have made great efforts in terms of technology and business solutions. All the exciting 4G/LTE base stations have been upgraded and the core networks were also optimized. These made the network more robust and covered larger areas. At the same time, each of them developed a variety of different billing methods which could keep the monthly fee at a very low level. They also established professional teams to provide technical support and formulated operator policy so that the service was more efficient and safer.

\section{Conclusion}

As a revolutionary and evolutionary regime of traditional grid, advanced metering infrastructure (AMI) has attracted rapidly growing attention in different areas. In this paper, we focused on reducing the deployment cost and prolonging network lifetime of AMI by introducing NB-IoT technology which could provide minimized power consumption and enhanced coverage. First, we presented a survey of AMI communication requirements mainly in terms of coverage range, data rate, and latency, and then studied the NB-IoT technology. Compared with the theoretical communication requirements of WAN/NAN/HAN, NB-IoT may be very suitable for some applications in HAN/NAN. Later, the five key technologies of NB-IoT were emphasized on. These could make this technology easier to understand and also help to achieve a more effective analysis of the performance in real-world NB-IoT network. Finally, the results from the aspects of coverage, reliability, latency, and battery life were presented, the performance was analyzed and discussed, and some options which can be used to improve performance were also proposed. These results demonstrated the potential of NB-IoT to support AMI. At the same time, the security issue of NB-IoT technology was also concerned. As a relatively new technology, NB-IoT may bring some worries to the customers, as discussed in the previous section, but they could be effectively and properly solved. It is foreseen that the new technology NB-IoT will be robust enough to support and boost AMI in smart grid.

\section{Abbreviations}

AMI: Advanced metering infrastructure; APNs: Access point names; CoAP: Constrained application protocol; DRX: Discontinuous reception; eDRX: 
Extended discontinuous reception; HAN: Home area network; LF: Low frequency; LPWANs: Low power wide area networks; M2M: Machine-to-machine; NAN: Neighborhood area network; NB: Narrowband; NB-IoT: Narrowband Internet of Things; NPBCH: Narrowband physical broadcast channel; NPDCCH: Narrowband physical downlink control channel; NPDSCH: Narrowband physical downlink shared channel; NRS: Narrowband reference signal; NPSS: Narrowband primary synchronization signals; NSSS: Narrowband secondary synchronization signals; NPRACH: Narrowband physical random access channel; NPUSCH: Narrowband physical uplink shared channel; OFDM: Orthogonal frequency-division multiplexing; PLR: Packet loss rate; PRB: Physical resource block; PSM: Power saving mode; PTW: Paging transmission window; RRC: Radio resource control; RSRP: Reference signal received power; SC-FDMA: Single-carrier frequency-division multiple access; SNR: Signal-to-noise ratio; SR: Signal repetition; TAU: tracking area update; UE: User equipment; WAN: Wide area network

\section{Acknowledgements}

This work was supported by State Key Laboratory of Smart Grid Protection and Control of NARI Group Corporation and The Key R\&D Program of Jiangsu Province industry prospect and common key technologies.

\section{Funding}

The funding was supported by State Key Laboratory of Smart Grid Protection and Control of NARI Group Corporation and The Key R\&D Program of Jiangsu Province industry prospect and common key technologies.

\section{Availability of data and materials}

Data sharing not applicable to this article as no datasets were generated during the current study.

\section{Authors' contributions}

LW and JW conceptualized the idea and designed the experiments. LW, ZJZ, and JW contributed to the writing and draft preparation, and JW supervised the research. All authors read and approved the final manuscript.

\section{Competing interests}

The authors declare that they have no competing interests.

\section{Publisher's Note}

Springer Nature remains neutral with regard to jurisdictional claims in published maps and institutional affiliations.

\section{Received: 2 August 2018 Accepted: 11 December 2018}

Published online: 07 January 2019

\section{References}

1. V. C. Gungor, D. Sahin, T. Kocak, et al., Smart grid technologies: communication technologies and standards. IEEE Trans. Ind. Inform. 7(4), 529-539 (2011)

2. NETL Modern Grid Strategy, Advanced metering infr astructure. US Dep. Energy Off. Electr. Energy Reliab, 1-34 (2008)

3. Z. Fan, P. Kulkarni, S. Gormus, et al., Smart grid communications: overview of research challenges, solutions, and standardization activities. IEEE Commun. Surv. Tutorials. 15(1), 21-38 (2013)

4. J. Zhang, A. Hasandka, J. Wei, et al., Hybrid communication architectures for distributed smart grid applications. Energies. 11(4), 871 (2018)

5. Electric Power Research Institute (EPRI), Advanced Metering Infrastructure (AMI) (2007). https://www.ferc.gov/CalendarFiles/20070423091846-EPRI \%20-\%20Advanced\%20Metering.pdf

6. J. Gao, Y. Xiao, J. Liu, et al., A survey of communication/networking in smart grids. Futur. Gener. Comput. Syst. 28(2), 391-404 (2012)

7. L. P. Qian, Y. J. A. Zhang, J. Huang, et al., Demand response management via real-time electricity price control in smart grids. IEEE J. Sel. Areas Commun. 31(7), 1268-1280 (2013)

8. J. Lloret, J. Tomas, A. Canovas, et al., An integrated loT architecture for smart metering. IEEE Commun. Mag. 54(12), 50-57 (2016)

9. R. R. Mohassel, A. Fung, F. Mohammadi, K. Raahemifar, A survey on advanced metering infrastructure. Electr. Power Energy Syst. 63(63), 473-484 (2014)

10. U. Raza, P. Kulkarni, M. Sooriyabandara, Low power wide area networks: A survey. arXiv preprint (2016)
11. R. Sanchez-Iborra, M.-D. Cano, State of the art in LP-WAN solutions for industrial loT services. Sensors. 16(5), 708 (2016)

12. VODAFONE Group PIc., New Study Item on Cellular System Support for Ultra Low Complexity and Low Throughput Internet of Things. (3GPP TSG-GERAN Meeting \#62, Valencia, 2014)

13. W. Wang, Y. Xu, M. Khanna, A survey on the communication architectures in smart grid. Comput. Netw. 55(15), 3604-3629 (2011)

14. M. Kuzlu, M. Pipattanasomporn, in Innovative Smart Grid Technologies (ISGT), 2013 IEEE PES. Assessment of communication technologies and network requirements for different smart grid applications (IEEE, 2013), pp. 1-6

15. X. Liu, M. Jia, X. Zhang, W. Lu, A novel multi-channel Internet of Things based on dynamic spectrum sharing in $5 \mathrm{G}$ communication. IEEE Internet Things J, 1-1 (2018)

16. Z. Na, J. Lv, F. Jiang, et al., Joint Subcarrier and Subsymbol Allocation based Simultaneous Wireless Information and Power Transfer for Multiuser GFDM in loT. IEEE Internet of Things J, 1-1 (2018)

17. X. Liu, M. Jia, Z. Na, et al., Multi-modal cooperative spectrum sensing based on dempster-shafer fusion in $5 \mathrm{G}$-based cognitive radio. IEEE Access. 6, 199-208 (2018)

18. J. Wang, C. Liu, W. Li, et al., Heterogeneous multi-mode access in smart grid using BeiDou communication. Microprocess. Microsyst. 47, 244-249 (2016)

19. N. Saputro, K. Akkaya, S. Uludag, A survey of routing protocols for smart grid communications. Comput. Netw. 56(11), 2742-2771 (2012)

20. R. R. Mohassel, A. S. Fung, F. Mohammadi, K. Raahemifar, in Electrical and Computer Engineering (CCECE), 2014 IEEE 27th Canadian Conference on. A survey on advanced metering infrastructure and its application in smart grids (IEEE, 2014), pp. 1-8

21. M. Kuzlu, M. Pipattanasomporn, S. Rahman, Communication network requirements for major smart grid applications in HAN, NAN and WAN. Comput. Netw. 67(10), 74-88 (2014)

22. A. Zaballos, A. Vallejo, J. M. Selga, Heterogeneous communication architecture for the smart grid. IEEE Netw. 25(5), 30-37 (2011)

23. H. Li, L. Lai, W. Zhang, Communication requirement for reliable and secure state estimation and control in smart grid. IEEE Trans. Smart Grid. 2(3), 476-486 (2011)

24. US DoE, Communications requirements of Smart Grid technologies. US Department of Energy, Tech. Rep, 1-69 (2010)

25. M. P. McHenry, Technical and governance considerations for advanced metering infrastructure/smart meters: technology, security, uncertainty, costs, benefits, and risks. Energy Policy. 59(3), 834-842 (2013)

26. R. Ratasuk, B. Vejlgaard, N. Mangalvedhe, et al., NB-loT system for M2M communication. Wirel. Commun. Netw. Conf. Workshops. IEEE. 59(3), 428-432 (2016)

27. Huawei, HiSilicon, TR 36.802 Narrowband Internet of Things (NB-IOT), Technical Report TR 36.802 V1.0.0. (3GPP TSG-RAN Meeting \#72, Busan, 2016)

28. J. Lee, J. Lee, Prediction-based energy saving mechanism in 3gpp nb-iot networks. Sensors. 17(9), 2008 (2017)

29. Y.-P. E. Wang, X. Lin, A. Adhikary, et al., A primer on 3GPP narrowband Internet of Things. IEEE Commun. Mag. 55(3), 117-123 (2017)

30. R. Ratasuk, N. Mangalvedhe, Y. Zhang, M. Robert, J.-P. Koskinen, in Standards for Communications and Networking (CSCN), 2016 IEEE Conference on. Overview of narrowband iot in Ite rel-13 (IEEE, 2016), pp. 1-7

31. Y. D. Beyene, R. Jäntti, O. Tirkkonen, et al., NB-loT technology overview and experience from cloud-RAN implementation. IEEE Wirel. Commun. 24(3), 26-32 (2017)

32. J. Schlienz, D. Raddino, Narrowband internet of things whitepaper. White Paper, Rohde\&Schwarz, 1-42 (2016)

33. M. Chen, Y. Miao, Y. Hao, et al., Narrow band internet of things. IEEE Access. 5(99), 20557-20577 (2017)

34. VODAFONE Group PIc (Rapporteur), TR 45.820 Cellular System Support for Ultra Low Complexity and Low Throughput Internet of Things (Release 13), v. 2.0.0. (3GPP GERAN Meeting \#67, Yinchuan, 2015)

35. L. Wan, Z. Zhang, J. Wang, et al., in International Conference on Computer Systems, Electronics and Control(ICCSEC),2017 IEEE Conference on. Performance Analysis of NB-IoT technology for Indoor loT Applications (IEEE, Dalian, 2017), pp. 2224-2228 